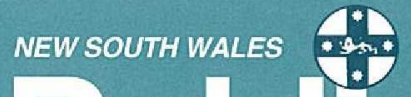 Public Health Bulletin
}

\section{Motivating local ACTION ON POOL DROWNINGS: A BLACK SPOT APPROACH FOR NSW}

Geoffrey Sayer and Cait Lonie

Injury Epidemiology Unit

Centre for Clinical Policy \& Practice

Public Health Division

NSW Health Department

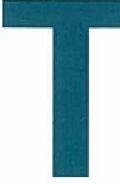

his article reports the most recent available NSW data on drowning and near-drowning of toddlers (aged 0-4 years) by Local Government Area. The data identify "black spots" and are intended to assist local government in determining priorities for remedial action.

\section{BACKGROUND}

NSW Health Goals and Targets for the prevention of drowning The drowning of toddlers (aged 0-4 years) in private swimming pools raises emotive debate and community conflict ${ }^{1}$. The NSW Health Goals and Targets identify preventing toddlers from drowning and preventing near-drowning incidents as priorities ${ }^{2}$. They propose a 50 per cent reduction in toddler drowning and near-drowning incidents by 2000 . Private swimming pools are the single most dangerous water environment for this age group. Drowning in swimming pools accounted for at least two-thirds of all drowning in the 0-4 age group during 1991-1993. The target would be achieved if all drownings in private swimming pools could be prevented.

The effective prevention of drowning requires monitoring of neardrowning incidents as well as deaths from drowning, but the causes of drowning and near-drowning may differ. Some toddlers involved in near-drowning incidents will have permission from adult supervisors to be in or around a pool, whereas most drownings occur after unintended access to a pool. Thus the major preventive actions available to reduce serious immersions are:

appropriate supervision of children who have permission
to be at the poolside; and
pool fencing to protect toddlers who make their way
unsupervised and without permission to a backyard pool.

\section{Legislation and regulation}

On August 1, 1992 the NSW Swimming Pools Act 1992 replaced legislation which had been passed in 1990. The new Act removed the retrospective requirement for all existing private pools to have isolation fencing. The legislation requires that any pool built after August 1, 1990 be surrounded by a child-resistant barrier that separates the pool from any residential building on the premises and from any place adjoining the premises. Pools built before that date must be surrounded by a child-resistant barrier that separates the pool from any adjoining premises.

\section{Contents}

\section{Articles}

139 Motivating local action on pool drownings: $a$ black spot approach for NSW

145 Incorporating evidence into clinical guidelines for the management of diabetes

147 Waiting List Reduction Program: results to

November 1995

151 Report of the NSW Midwives Data Collection 1994

\section{Infectious Diseases}

152 Notification trends

\section{Tables}

\section{Correspondence}

Please address all correspondence and potential contributions to:

The Editor,

NSW Public Health Bulletin,

Public Health Division,

NSW Health Department

Locked Bag No 961,

Nortb Sydney NSW 2059

Telepbone: (02) 3919191

Facsimile: (02) 3919029 


\section{Local action on pool drownings}

Continued from page 139

The Pool Fencing Advisory Committee was set up under the Swimming Pools Act 1992 within the Department of Local Government and Co-operatives. The committee is to consider data on drowning and near-drowning, both past and present, analyse the data and advise and make recommendations to the Minister on appropriate amendments to the Act regarding fencing legislation.

The committee has recommended that:

- a single, agreed minimum set of standard data be collected on all private pool drowning and neardrowning in NSW;

- a regular survey or inspection program of private pools be undertaken to collect information on the number of private pools, including data on fencing configuration, pool owner and frequency of child visitors to the residence; and a special study be supported to provide definitive answers concerning the protective value of pool fencing ${ }^{3}$.

Local councils are responsible for regulation and for the enforcement of the Act. There has been no statewide evaluation of the effectiveness of the legislation and no assessment of where the most serious immersions take place or where toddlers are at greatest risk.

\section{METHOD}

Data sources for this study comprised:

- the NSW Inpatient Statistics Collection (ISC) for the 1986 calendar year and financial years 1988-891993-94; and

- Australian Bureau of Statistics (ABS) mortality data for 1979-1992.

Only incidents classified as swimming pool immersions (E910.8 in the International Classification of Diseases and Causes of Death, version 9, Clinical Modification) were included. Indirect standardised rates were calculated to allow for changes in population structure over the period studied, with 1991-92 taken as the reference year for ISC data and 1991 for ABS mortality data.

Because pool immersion incidents in Local Government Areas (LGAs) are rare, it was necessary to combine data from several years to assess possible changes from a baseline. Deaths and hospital separations from 1986 and 1989-92 were combined to produce rates of serious immersions (i.e. near-drownings) of toddlers. To avoid double counting of a serious immersion, patients who subsequently died or were transferred from another hospital were removed from the ISC data. The remaining records from deaths and ISC data were considered a serious immersion in a swimming pool (SI).

Indirectly standardised serious immersion ratios (SSIR) were calculated for each LGA. The standardisation of the data was adjusted for differences in the sex distribution (there was only one age group under examination) between the State population and the LGA population. Conventional 99 per cent confidence intervals were calculated for each

\section{FIGURE 1}

DEATHS BY IMMERSION IN SWIMIMING POOLS

BY 0-4 YEAR OLDS, NSW, 1979-1992

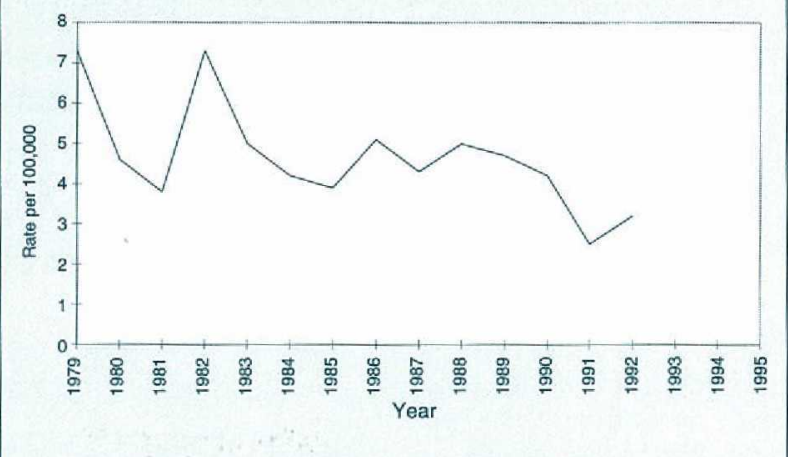

Source: Australian Bureau of Statistics: ICD9-CM E910.8

\section{FIGURE 2}

NSW HOSPITAL SEPARATIONS FOR IMMERSIONS IN SWIMIMING POOLS FOR 0-4 YEAR OLDS, 1986 - 1993-94

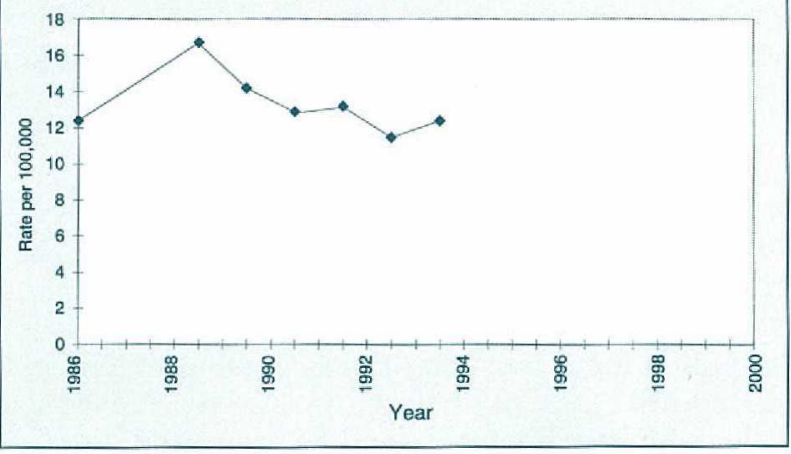

Source: Inpatient Statistics Collection, ICD9-CM E910.8'

1. Separations as a result of death are also included.

SSIR based on the Normal Approximation Method, and the SSIR for the State as a whole was taken as 100 (thus an LGA with a SSIR of 300 would have a rate three times the State average).

\section{RESULTS}

There was a marked reduction in mortality associated with drowning between 1979 and 1992. The numbers of deaths of toddlers from drowning in swimming pools declined from 29 $(7.3 / 100,000)$ in 1979 to $14(3.2 / 100,000)$ in 1992 (Figure 1).

By contrast, there was little change in the incidence of neardrowning among toddlers in swimming pools between 1986 and 1993-94, as indicated by hospital separations for immersion recorded in the ISC between 1986 and in 199394 (Figure 2).

Table 1 lists the cumulative SSIR for each LGA over the period 1986 and 1989-1992. In many LGAs no SI incidents 


\section{TABLE 1}

STANDARDISED SERIOUS IMMERSION RATIOS BY

LOCAL GOVERNMENT AREA, NSW, 1986, 1989-1992

\begin{tabular}{|c|c|c|c|c|c|c|}
\hline LGA & SI & EXP SI & SSIR & SSIR SE & LCI99 & UCl99 \\
\hline Albury & 1 & 2.6802 & 37.311 & 37.311 & 0.0 & 133.57 \\
\hline Armidale & - & 1.3035 & - & - & - & - \\
\hline Ashfield & - & 1.9043 & - & - & - & - \\
\hline Auburn & 2 & 3.2196 & 62.119 & 43.925 & 0.0 & 175.44 \\
\hline Ballina & 1 & 1.5397 & 64.948 & 64.948 & 0.0 & 232.51 \\
\hline Balranald & - & 0.2115 & - & - & - & - \\
\hline Bankstown & 8 & 8.8485 & 90.411 & 31.965 & 7.94 & 172.88 \\
\hline Barraba & - & 0.1502 & - & - & - & - \\
\hline Bathurst, Evans and Oberon & 1 & 2.3839 & 41.950 & 41.950 & 0.0 & 150.18 \\
\hline Baulkham Hills & 5 & 6.1680 & 81.063 & 36.253 & 0.0 & 174.60 \\
\hline Bega Valley & 1 & 1.7942 & 55.734 & 55.734 & 0.0 & 199.53 \\
\hline Bellingen & - & 0.8215 & - & - & - & - \\
\hline Berrigan & 1 & 0.5244 & 190.707 & 190.707 & 0.0 & 682.73 \\
\hline Bingara & - & 0.1280 & - & - & & - \\
\hline Bland & 1 & 0.4919 & 203.285 & 203.285 & $0 . \overline{0}$ & 727.76 \\
\hline Blue Mountains & 4 & 4.8361 & 82.712 & 41.356 & 0.0 & 189.41 \\
\hline Bogan & - & 0.2414 & - & - & - & - \\
\hline Bombala & - & 0.2085 & - & - & - & - \\
\hline Boorowa & - & 0.1707 & - & - & - & - \\
\hline Botany & 2 & 1.8212 & 109.816 & 77.651 & 0.0 & 310.16 \\
\hline Bourke & - & 0.3966 & - & - & - & - \\
\hline Brewarrina & - & 0.2337 & - & - & - & - \\
\hline Broken Hill & 1 & 1.5136 & 66.067 & 66.067 & 0.0 & 236.52 \\
\hline Burwood & 1 & 1.3756 & 72.694 & 72.694 & 0.0 & 260.24 \\
\hline Byron & 1 & 1.4620 & 68.399 & 68.399 & 0.0 & 244.87 \\
\hline Camden & 5 & 1.5203 & 328.883 & 147.081 & 0.0 & 708.35 \\
\hline Campbelltown & 13 & 12.5347 & 103.712 & 28.765 & 29.50 & 177.92 \\
\hline Canterbury & 2 & 8.1573 & 24.518 & 17.337 & 0.0 & 69.25 \\
\hline Carrathool & - & 0.2541 & - & - & - & - \\
\hline Casino & - & 0.7091 & - & - & - & - \\
\hline Central Darling & 1 & 0.2561 & 390.459 & 390.459 & 0.0 & $1,397.84$ \\
\hline Cessnock & 3 & 2.9507 & 101.669 & 58.699 & 0.0 & 253.11 \\
\hline Cobar & - & 0.4558 & - & - & - & 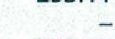 \\
\hline Coffs Harbour & 4 & 3.1586 & 126.637 & 63.318 & 0.0 & 290.00 \\
\hline Conargo & - & 0.1060 & - & - & - & - \\
\hline Concord & 1 & 1.1636 & 85.940 & 85.940 & 0.0 & 307.67 \\
\hline Coolah & - & 0.2663 & - & - & - & - \\
\hline Coolamon & - & 0.2762 & - & - & - & - \\
\hline Cooma-Monaro & - & 0.6380 & - & - & - & -1 \\
\hline Coonabarabran & 2 & 0.4923 & 406.267 & 287.274 & 0.0 & $1,147.43$ \\
\hline Coonamble & - & 0.4236 & - & - & - & - \\
\hline Cootamundra & - & 0.5467 & - & - & - & - \\
\hline Copmanhurst & - & 0.2601 & - & - & - & - \\
\hline Corowa & - & 0.4560 & - & - & - & - \\
\hline Cowra & 1 & 0.7994 & 125.087 & 125.087 & 0.0 & 447.81 \\
\hline Crookwell & - & 0.3106 & - & - & - & - \\
\hline Culcairn & - & 0.2685 & - & - & - & - \\
\hline Deniliquin & - & 0.5413 & - & - & - & 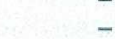 \\
\hline Drummoyne & - & 1.4089 & - & - & - & - \\
\hline Dubbo & 4 & 2.5029 & 159.817 & 79.908 & 0.0 & 365.98 \\
\hline Dumaresq & - & 0.2376 & - & - & - & - \\
\hline Dungog & - & 0.4732 & - & - & - & - \\
\hline Eurobodalla & - & 1.5381 & - & - & - & $\therefore-$ \\
\hline Fairfield & 13 & 12.0454 & 107.925 & 29.933 & 30.70 & 185.15 \\
\hline Forbes & - & 0.6796 & - & - & - & - \\
\hline Gilgandra & - & 0.3443 & - & - & - & - \\
\hline Glen Innes & - & 0.39624 & - & - & - & - \\
\hline Gloucester & 1 & 0.29687 & 336.847 & 336.847 & 0.0 & $1,205.91$ \\
\hline Gosford & 9 & 8.22751 & 109.389 & 36.463 & 15.31 & 203.46 \\
\hline Goulburn & 1 & 1.37627 & 72.660 & 72.660 & 0.0 & 260.12 \\
\hline Grafton & - & 1.06051 & - & - & - & - \\
\hline Greater Lithgow & 1 & 1.35315 & 73.902 & 73.902 & 0.0 & 264.57 \\
\hline Taree and Great Lakes & 2 & 3.83812 & 52.109 & 36.847 & 0.0 & 147.17 \\
\hline Griffith & 1 & 1.54382 & 64.774 & 64.774 & 0.0 & 231.89 \\
\hline Gundagai & - & 0.27718 & - & - & - & - \\
\hline Gunnedah & 1 & 0.95510 & 104.701 & 104.701 & 0.0 & 374.83 \\
\hline
\end{tabular}




\section{TABLE 1 (Continued)}

STANDARDISED SERIOUS IMMERSION RATIOS BY

LOCAL GOVERNMENT AREA, NSW, 1986, 1989-1992

\begin{tabular}{|c|c|c|c|c|c|c|}
\hline LGA & SI & EXP SI & SSIR & SSIR SE & LCl99 & UCl99 \\
\hline Gunning & - & 0.15367 & - & - & - & - \\
\hline $\begin{array}{l}\text { Guyra } \\
\text { Harden }\end{array}$ & - & $\begin{array}{l}0.35155 \\
078235\end{array}$ & - & $\begin{array}{l}- \\
-\end{array}$ & - & - \\
\hline $\begin{array}{l}\text { Harden } \\
\text { Hastings }\end{array}$ & $\overline{1}$ & $\begin{array}{l}0.28235 \\
2.67231\end{array}$ & $37.42 \overline{1}$ & 37.421 & $0 . \overline{0}$ & 133.97 \\
\hline $\begin{array}{l}\text { Hastings } \\
\text { Hawkesbury }\end{array}$ & 5 & $\begin{array}{l}2.67231 \\
4.09056\end{array}$ & 122.233 & 54.664 & 0.0 & 263.27 \\
\hline Hay & - & 0.27803 & - & - & - & - \\
\hline Holbrook & - & 0.18276 & - & - & - & - \\
\hline Holroyd & 6 & 4.81057 & 124.725 & 50.919 & 0.0 & 256.10 \\
\hline Hornsby & 10 & 7.49788 & 133.371 & 42.176 & 24.56 & 242.18 \\
\hline $\begin{array}{l}\text { Hume } \\
\text { Hunter's Hill }\end{array}$ & - & 0.44031 & רา & - & - & - \\
\hline $\begin{array}{l}\text { Hunter's Hill } \\
\text { Hurstville }\end{array}$ & 4 & 0.47912 & 834.872 & 417.436 & 0.0 & $1,911.86$ \\
\hline $\begin{array}{l}\text { Hurstville } \\
\text { Inverell }\end{array}$ & 2 & 3.22824 & 61.953 & 43.808 & 0.0 & 174.98 \\
\hline $\begin{array}{l}\text { Inverell } \\
\text { Jerilderie }\end{array}$ & & $\begin{array}{l}1.06084 \\
0.13562\end{array}$ & - & - & - & - \\
\hline Junee & $\overline{1}$ & $\begin{array}{l}0.13562 \\
0.37276\end{array}$ & $268.27 \overline{0}^{-}$ & 268.270 & $\overline{0}$ & - \\
\hline Kempsey & 3 & $\begin{array}{l}0.37276 \\
1.68985\end{array}$ & $\begin{array}{l}268.270 \\
177.530\end{array}$ & $\begin{array}{l}268.270 \\
102.497\end{array}$ & 0.0 & 960.41 \\
\hline Kiama & - & $\begin{array}{l}1.68985 \\
1.03820\end{array}$ & $\begin{array}{r}177.530 \\
-\end{array}$ & $\begin{array}{r}102.497 \\
-\end{array}$ & $\begin{array}{r}0.0 \\
-\end{array}$ & 441.97 \\
\hline Kogarah & 2 & 2.24460 & $89.10 \overline{3}$ & 63.005 & $0 . \overline{0}$ & 2515 \\
\hline Ku-ring-gai & 19 & 4.22498 & 449.706 & $\begin{array}{r}63.005 \\
103.170\end{array}$ & $\begin{array}{r}0.0 \\
183.53\end{array}$ & $\begin{array}{l}251.66 \\
715.88\end{array}$ \\
\hline Lachlan & - & 0.60387 & - & $\begin{array}{r}103.1 / 0 \\
-\end{array}$ & $\begin{array}{r}183.53 \\
-\end{array}$ & $\begin{array}{r}715.88 \\
-\end{array}$ \\
\hline Lake Macquarie & 13 & 9.99383 & 130.080 & 36.078 & 37.000 & $223.1 \overline{-}$ \\
\hline Lane Cove & 2 & 1.35376 & 147.737 & 104.466 & 0.0 & 417.26 \\
\hline Leeton & 1 & 0.74351 & 134.496 & 134.496 & 0.0 & 481.50 \\
\hline Leichhardt & $\overline{-}$ & 2.66002 & - & - & - & - \\
\hline Lismore and Kyogle & 2 & 3.33978 & 59.884 & 42.345 & 0.0 & 169.13 \\
\hline Liverpool & 15 & 6.95351 & 215.719 & 55.698 & 72.02 & 359.42 \\
\hline Lockhart & 2 & 0.27016 & 740.301 & 523.472 & 0.0 & $2,090.86$ \\
\hline Maclean & 1 & 0.72687 & 137.576 & 137.576 & 0.0 & 492.52 \\
\hline Maitland & 3 & 3.35932 & 89.304 & 51.560 & 0.0 & 222.33 \\
\hline Manilla & - & 0.20894 & - & - & - & - \\
\hline Manly & 3 & 1.65224 & 181.571 & 104.830 & 0.0 & 452.03 \\
\hline Marrickville & 2 & 4.24056 & 47.164 & 33.350 & 0.0 & 133.21 \\
\hline Merriwa & - & 0.16872 & - & - & - & - \\
\hline $\begin{array}{l}\text { Moree Plains } \\
\text { Mosman }\end{array}$ & 1 & 1.39580 & 71.644 & 71.644 & 0.0 & 256.48 \\
\hline $\begin{array}{l}\text { Mosman } \\
\text { Mudgee }\end{array}$ & $\overline{1}$ & 1.10127 & - & - & - & 25 \\
\hline $\begin{array}{l}\text { Mudgee } \\
\text { Mulwaree }\end{array}$ & 1 & 1.14282 & 87.503 & 87.503 & 0.0 & 313.26 \\
\hline $\begin{array}{l}\text { Mulwaree } \\
\text { Murray }\end{array}$ & & 0.36092 & - & - & - & - \\
\hline Murrumbidgee & - & $\begin{array}{l}0.31277 \\
0.17532\end{array}$ & - & $\begin{array}{l}- \\
-\end{array}$ & - & - \\
\hline Murrurundi & - & $\begin{array}{l}0.17532 \\
0.15325\end{array}$ & - & - & - & - \\
\hline Muswellbrook & 1 & $\begin{array}{l}0.15325 \\
1.20935\end{array}$ & 82.689 & 82.689 & $0 . \overline{0}$ & $296.0 \overline{3}$ \\
\hline Nambucca & 2 & $\begin{array}{l}1.20935 \\
1.04373\end{array}$ & 191.620 & 135.495 & 0.0 & $\begin{array}{l}296.03 \\
541.20\end{array}$ \\
\hline Narrabri & 3 & 1.06330 & 282.139 & 162.893 & 0.0 & $\begin{array}{l}541.20 \\
702.40\end{array}$ \\
\hline Narrandera & - & 0.49983 & - & - & - & $\begin{array}{r}702.40 \\
-\end{array}$ \\
\hline Narromine & - & 0.50334 & - & - & - & - \\
\hline Newcastle & 9 & 6.89363 & 130.555 & 43.518 & 18.28 & 242.83 \\
\hline North Sydney & 1 & $\begin{array}{r}1.4947 \\
-\quad 10\end{array}$ & 66.902 & 66.902 & 0.0 & $\begin{array}{l}242.83 \\
239.51\end{array}$ \\
\hline Nundle & - & 0.0836 & - & - & - & 239.51 \\
\hline Nymboida & 2 & 0.3033 & 659.404 & 466.269 & 0.0 & $1,862.38$ \\
\hline Orange, Blayney and Cabonne & 3 & 3.4488 & 86.987 & 50.222 & 0.0 & $\begin{array}{r}1,862.38 \\
216.56\end{array}$ \\
\hline Parkes & - & 0.9773 & - & - & - & $\begin{array}{r}216.56 \\
-\end{array}$ \\
\hline Parramatta and Blacktown & 20 & 24.1454 & 82.832 & 18.522 & 35.05 & $130 . \overline{2}^{-}$ \\
\hline Parry & - & 0.7708 & - & - & - & $\begin{array}{r}130.62 \\
-\end{array}$ \\
\hline Penrith & 20 & 12.3338 & 162.156 & 36.259 & 68.61 & $255 . \overline{70}$ \\
\hline Port Stephens & 1 & 3.0208 & 33.104 & 33.104 & 0.0 & 118.51 \\
\hline Queanbeyan & 2 & 1.7682 & 113.112 & 79.982 & 0.0 & 319.47 \\
\hline Quirindi & $\bar{r}$ & 0.3502 & - & - & - & - \\
\hline Randwick & 5 & 4.8066 & 104.024 & 46.521 & 0.0 & 224.05 \\
\hline Richmond River & $\overline{-}$ & 0.5811 & - & - & - & - \\
\hline Rockdale & 3 & 4.1492 & 72.304 & 41.745 & 0.0 & 180.00 \\
\hline Ryde & 4 & 4.8262 & 82.881 & 41.440 & 0.0 & 189.80 \\
\hline Rylstone & - & 0.2545 & - & . & - & - \\
\hline Scone & 1 & 0.6700 & 149.265 & 149.265 & 0.0 & 534.37 \\
\hline Severn & $\overline{7}$ & 0.1982 & - & - & - & - \\
\hline Shellharbour & 7 & 3.3704 & 207.688 & 78.499 & 5.16 & 410.21 \\
\hline Shoalhaven & 3 & 4.4998 & 66.670 & 38.492 & 0.0 & 165.98 \\
\hline
\end{tabular}




\section{TABLE 1 (Continued)}

STANDARDISED SERIOUS IMMERSION RATIOS BY

LOCAL GOVERNMENT AREA, NSW, 1986, 1989-1992

\begin{tabular}{|c|c|c|c|c|c|c|}
\hline LGA & SI & EXP SI & SSIR & SSIR SE & LCl99 & UCI99 \\
\hline Singleton & 2 & 1.4171 & 141.137 & 99.799 & 0.0 & 398.62 \\
\hline Snowy River & - & 0.3156 & 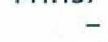 & 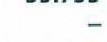 & - & - \\
\hline Strathfield & - & 1.1850 & - & - & - & - \\
\hline Sutherland & 10 & 11.3625 & 88.009 & 27.831 & 16.21 & 159.81 \\
\hline Tallaganda & - & 0.1535 & - & - & - & - \\
\hline Tamworth & 2 & 2.3210 & 86.170 & 60.931 & 0.0 & 243.37 \\
\hline Temora & 1 & 0.4399 & 227.339 & 227.339 & 0.0 & 813.87 \\
\hline Tenterfield & 1 & 0.4315 & 231.773 & 231.773 & 0.0 & 829.75 \\
\hline Tumbarumba & - & 0.2636 & - & - & - & - \\
\hline Tumut & - & 0.7766 & - & - & - & - \\
\hline Tweed & 7 & 3.1256 & 223.957 & 84.647 & 5.57 & 442.35 \\
\hline Ulmarra & 2 & 0.4230 & 472.850 & 334.356 & 0.0 & $1,335.49$ \\
\hline Uralla & - & 0.4113 & - & - & - & - \\
\hline Urana & - & 0.1148 & - & - & - & - \\
\hline Wagga Wagga & 3 & 3.7499 & 80.001 & 46.189 & 0.0 & 199.17 \\
\hline Wakool & - & 0.3558 & - & - & - & - \\
\hline Walcha & - & 0.2407 & - & - & - & - \\
\hline Walgett & 1 & 0.5932 & 168.576 & 168.576 & 0.0 & 603.50 \\
\hline Warren & - & 0.2735 & - & - & - & - \\
\hline Warringah & 10 & 8.8529 & 112.958 & 35.720 & 20.80 & 205.12 \\
\hline Waverley & - & 2.4620 & - & 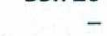 & - & - \\
\hline Weddin & - & 0.2726 & - & - & - & - \\
\hline Wellington & 2 & 0.6559 & 304.907 & 215.602 & 0.0 & 861.16 \\
\hline Wentworth & 1 & 0.4731 & 211.378 & 211.378 & 0.0 & 756.73 \\
\hline Willoughby & 1 & 2.5954 & 38.529 & 38.529 & 0.0 & 137.93 \\
\hline Windouran & - & 0.0293 & - & - & - & - \\
\hline Wingecarribee & - & 2.2409 & - & - & - & - \\
\hline Wollondilly & 2 & 2.4368 & 82.074 & 58.035 & 0.0 & 231.80 \\
\hline Wollongong & 11 & 10.5743 & 104.026 & 31.365 & 23.10 & 184.95 \\
\hline Woollahra & - & 1.6555 & - & 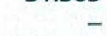 & - & - \\
\hline Wyong & 8 & 6.6564 & 120.185 & 42.492 & 10.56 & 229.81 \\
\hline Yallaroi & 2 & 0.2434 & 821.585 & 580.948 & 0.0 & $2,320.43$ \\
\hline Yarrowlumla & - & 0.5386 & - & - & - & - \\
\hline Young & 1 & 0.7152 & 139.829 & 139.829 & 0.0 & 500.59 \\
\hline
\end{tabular}

Abbreviations

LGA = Local Government Area

$\mathrm{SI}=$ Serious Immersions (includes deaths and hospital separations)

Exp $\mathrm{SI}=$ Expected number of serious immersions

SSIR = Standardised Serious Immersion Ratio

SSIR SE = Standardised Serious Immersion Ratio Standard Error

LCI $99=$ Lower $99 \%$ confidence interval

UCI99 = Upper $99 \%$ confidence interval

\section{TABLE 2}

SERIOUS IMMERSIONS FOR 0-4 YEARS BY

LOCAL GOVERNMENT AREA, NSW, 1986, 1989-1993

\begin{tabular}{|c|c|c|c|c|c|c|c|c|}
\hline \multirow[b]{2}{*}{ Local Government } & \multicolumn{4}{|c|}{$1986-92 *$} & \multicolumn{4}{|c|}{1993} \\
\hline & SI & Exp SI & Total Pop & Rate§ & SI & Exp SI & Pop & Rate \\
\hline Campbelltown & 13 & 12.5 & 76,636 & 17 & 2 & 1.9 & 15,398 & 13 \\
\hline Canterbury & 2 & 8.2 & 49,839 & 4 & - & 1.2 & 10,052 & - \\
\hline Gosford & 9 & 8.2 & 49,895 & 18 & 1 & 1.3 & 10,684 & 9.4 \\
\hline Ku-ring-gai & 19 & 4.2 & 25,984 & 73 & - & 0.6 & 4,941 & - \\
\hline Parramatta/Blacktown+ & 20 & 24.0 & 146,492 & 14 & 2 & 3.8 & 30,674 & 6.5 \\
\hline Lake Macquarie & 13 & 10.0 & 61,160 & 21 & 4 & 1.5 & 12,330 & 32.4 \\
\hline Marrickville & 2 & 4.2 & 26,075 & 8 & - & 0.6 & 5,054 & 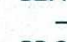 \\
\hline Penrith & 20 & 12.3 & 75,843 & 26 & 5 & 1.8 & 14,728 & 33.9 \\
\hline Sutherland & 10 & 11.2 & 68,468 & 15 & 1 & 1.7 & 14,015 & 7.1 \\
\hline Tweed & 7 & 3.1 & 18,246 & 38 & 2 & 0.5 & 4,049 & 49.4 \\
\hline Warringah & 10 & 8.9 & 54,293 & 18 & 4 & 1.3 & 10,711 & 37.3 \\
\hline Wyong & 8 & 6.7 & 39,687 & 20 & 5 & 1.1 & 8,907 & 56.1 \\
\hline State total & 346 & 346 & $2,120,865$ & 16 & 54 & 54 & 436,497 & 12.4 \\
\hline
\end{tabular}

$\mathrm{SI}=$ Serious Immersion (includes deaths and hospital separations), Exp $\mathrm{SI}=$ Expected number of serious immersions, Pop $=$ Population

* No data from 1987 and 1988 were used due to incomplete Inpatient Statistics Collection.

${ }^{5}$ Crude rate per 100,000 population.

+ Parramatta and Blacktown are combined because of boundary changes. 


\section{Local action on pool drownings}

\section{Continued from page 140}

occurred during this period. Of those where at least one SI occurred, Canterbury had the lowest incidence in relation to the State average. Only Ku-ring-gai LGA had a significantly higher rate than the State average, with 15 more incidents than expected over five years.

SIs in 13 LGAs were examined in more detail (Table 2). These LGAs were selected because of the number of SIs that occurred in them, or because they had substantial populations aged 0-4 years. During 1986-1992 these LGAs accounted for 35 per cent of all SIs and 33 per cent of the State's population aged 0-4 years. Although death data for 1993 were possibly incomplete, there were higher numbers of SIs than expected in the Lake Macquarie, Penrith, Tweed, Wyong and Warringah LGAs.

The Ku-ring-gai area averaged about three SIs a year in 1986-1992 - similar to the number of SIs in Parramatta and Blacktown LGAs, which had a combined population 5-6 times larger. There were no SIs in the Ku-ring-gai LGA in 1993, while there were five SIs in the Wyong LGA - twice as many as in the Parramatta LGA but with less than onethird the population aged 0-4 years.

\section{Discussion}

The differences among LGAs in SI rates may have resulted from several factors, including differences in the numbers of pools among LGAs (and therefore the amount of exposure), and variations in fencing configurations or the degree to which the legislation was enforced. Unfortunately, we do not have sufficient information to confirm or refute these possibilities.

The objective of this report was to identify "black spots" LGAs with an excess of serious immersions of toddlers which could motivate local prevention efforts. These efforts could complement (not replace) Statewide campaigns aimed at increasing compliance with pool fencing legislation and parental education about cardiopulmonary resuscitation, the causes of serious immersions and the need for vigilance.

The concept of statistical significance with 99 per cent confidence may be inappropriate when the number of incidents in a local area is few. Lower levels of confidence may be more acceptable and appropriate to determine possible black spots.
Alternatively, if the State target of a 50 per cent reduction from a baseline rate is considered acceptable, an assessment of the number of events in excess of this rate may provide a practical approach. However, if there is a community demand to prevent all drownings or near-drowning incidents in swimming pools in the 0-4 age group, every event should be investigated at a local level.

The Mental Health Epidemiology Group has suggested this approach in relation to suicides. It involves local auditing of the events that led to each suicide death or attempt, with an emphasis on immediate local action, rather than waiting for sufficient numbers of incidents to occur for statistical analysis. Auditing is defined as the collecting of information to promote or enforce positive change. Timeliness is an essential component of any audit process.

There is no adequate process of auditing serious immersions at a local level. As recommended by the Pool Fencing Advisory Committee ${ }^{3}$, the establishment of a drowning and near-drowning register that led to an immediate response, an assessment of the factors and events leading up to the incident and subsequent action at local and State levels in response to the contributing factors, would improve our understanding of the causes of serious immersions and increase the likelihood of reducing their number.

The information to be collected on private pools, also recommended by the Pool Fencing Advisory Committee ${ }^{3}$, would help determine the reasons for the variations in rates among LGAs. A review of local government approaches to the enforcement of pool fencing legislation could be related to population rates over time, and this could help to explain the varying temporal trends.

\section{ACKNOWLEDGMENT}

We thank David Lyle for his advice and assistance.

1. Carey V, Chapman S, Gaffney D. Children's lives or garden aesthetics? A case study in public health advocacy. Aust J of Public Health 1994; 18:25-32.

2. New South Wales Health Department. New South Wales Goals and Targets for Injury. March 1995.

3. Pool Fencing Advisory Committee. Monitoring of Serious Immersions in Private Pools in New South Wales. April 1994.

4. Chipps J, Stewart G, Sayer G. Suicide mortality in NSW: An introduction to the clinical audit. NSW Public Health Bulletin 1995; 7:68-70.

\section{PUBLIC HEALTH EDITORIAL STAFF}

The editor of the Public Health Bulletin is Dr Michael Frommer, Director, Research and Development, NSW Health Department. Dr Lynne Madden is production manager.

The Bulletin aims to provide its readers with population health data and information to motivate effective public health action. Articles, news and comments should be 1,000 words or less in length and include a summary of the key points to be made in the first paragraph. References should be set out using the Vancouver style, the full text of which can be found in British Medical Journal 1988; 296:401-5.

Please submit items in hard copy and on diskette, preferably using WordPerfect, to the editor, NSW Public Health Bulletin, Locked Mail Bag 961, North Sydney 2059. Facsimile (02) 3919029.

Please contact your local Public Health Unit to obtain copies of the NSW Public Health Bulletin. 